\title{
ILEEM-survey on the Heart Team approach and team training for lead extraction procedures
}

Christoph T. Starck ${ }^{1,2,3}$, Frank Bracke ${ }^{4}$, Peter-Paul Delnoy ${ }^{5}$, Roger A. Freedman ${ }^{6}$, Andrzej Kutarski ${ }^{7}$, Mark Gallagher ${ }^{8}$, Morio Shoda ${ }^{9,10}$, Robert Peyton ${ }^{11}$, Manav Sohal ${ }^{8}$, Frederik Gadler $^{12}$, Kamil Sedlacek ${ }^{13}$, Juha Hartikainen ${ }^{14}$, Patrizio Mazzone ${ }^{15}$, Alexander Breitenstein ${ }^{16}$, Nigel Lever ${ }^{17}$

${ }^{1}$ German Heart Center Berlin, Department of Cardiothoracic and Vascular Surgery, Berlin, Germany;

${ }^{2}$ German Center of Cardiovascular Research (DZHK), partner site Berlin, Berlin, Germany;

${ }^{3}$ Steinbeis University Berlin, Institute (STI) of Cardiovascular Perfusion, Berlin, Germany; ${ }^{4}$ Department of Cardiology, Catharina Hospital, Eindhoven, The Netherlands; ${ }^{5}$ Department of Cardiology, Isala Hospital,

Zwolle, The Netherlands; ${ }^{6}$ University of Utah Sciences Center, Salt Lake City, Utah, United States;

${ }^{7}$ Department of Cardiology, Medical University of Lublin, Poland; ${ }^{8}$ Cardiology Clinical Academic Group,

St. George's University Hospitals NHS Foundation Trust, London, United Kingdom; ${ }^{9}$ Department of

Cardiology, Tokyo Women's Medical University, Tokyo, Japan; ${ }^{10}$ Department of Cardiovascular Medicine,

Shinshu University School of Medicine, Matsumoto, Japan; ${ }^{11}$ UNC REX Healthcare, Cardiac Surgical

Specialists, Raleigh, North Carolina, United States; ${ }^{2}$ Department of Cardiology, Karolinska University

Hospital, Stockholm, Sweden; ${ }^{13}$ Department for Cardiology, Institute for Clinical and Experimental Medicine

(IKEM), Prague, Czech Republic; ${ }^{14}$ Heart Center, Kuopio University Hospital and University of Eastern

Finland, Kuopio, Finland; ${ }^{15}$ Department of Arrhythmology and Cardiac Pacing Unit, IRCCS Ospedale

San Raffaele, Milan, Italy; ${ }^{16}$ Division of Electrophysiology and Pacing, Department of Cardiology,

University Heart Center Zurich, Switzerland; ${ }^{17}$ Green Lane Cardiovascular Service,

Auckland City Hospital, and University of Auckland, New Zealand

\begin{abstract}
Background: The Heart Team approach has become an integral part of modern cardiovascular medicine. To evaluate current opinions and real-world practice among lead extraction practitioners, an online survey was created and distributed among a pool of lead extraction specialists participating in the International Lead Extraction Expert Meeting (ILEEM) 2018.

Methods: The online survey consisted of 10 questions and was performed using an online survey tool (www.surveymonkey.com). The collector link was sent to 48 lead extraction experts via email.

Results: A total of 43 answers were collected (89\% return rate) from lead extraction experts in 16 different countries. A great majority (83.7\%) of the respondents performed more than 30 lead extraction procedures per year. The most common procedural environment in this survey was the hybrid operating room (67.4\%). Most procedures were performed by electrophysiologists and cardiologists (80.9\%). Important additional members of the current lead extraction teams were cardiac surgeons (79.1\%), anesthesiologists (95.3\%) and operating room scrub nurses (76.7\%). An extended Heart Team is regarded beneficial for patient care by $86.0 \%$, with potential further members being infectious diseases specialists, intensivists and radiologists. Team training activities are performed in $48.8 \%$ of participating centers. Conclusions: This survey supports the importance of establishing lead extraction Heart Teams in specialized lead extraction centers to potentially improve patient outcomes. The concept of a core and an extended Heart Team approach in lead extraction procedures is introduced. (Cardiol J 2022; 29, 3: 481-488)

Key words: lead extraction, Heart Team, team training, pacemaker, ICD
\end{abstract}

Address for correspondence: Prof. Dr. Christoph T. Starck, MD, FEHRA, Department of Cardiothoracic and Vascular Surgery, German Heart Center Berlin, Augustenburger Platz 1, 13353 Berlin, Germany, tel: +49 3045932055 , e-mail: starck@dhzb.de

Received: 29.10.2019 Accepted: 21.07.2020 Early publication date: 14.08.2020

This article is available in open access under Creative Common Attribution-Non-Commercial-No Derivatives 4.0 International (CC BY-NC-ND 4.0) license, allowing to download articles and share them with others as long as they credit the authors and the publisher, but without permission to change them in any way or use them commercially. 


\section{Introduction}

The Heart Team approach plays an important role in modern cardiovascular medicine. The main purpose of the Heart Team is to determine the best available therapy in an individual patient using a multidisciplinary team approach, balancing the risks and benefits of different therapeutic strategies. The implementation of a multidisciplinary team approach has been recommended in multiple European Society of Cardiology guidelines (e.g. management of valvular disease, myocardial revascularization, management of atrial fibrillation, heart failure and infective endocarditis) [1-5].

For transvenous lead extraction (TLE) procedures the multidisciplinary team approach is noted in section 11.1 ("Personnel") of the current 2017 Heart Rhythm Society expert consensus statement. As members of this multidisciplinary team, cardiologists, electrophysiologists, cardiothoracic surgeons (in centers where the primary operator is not a surgeon), interventional radiologists, vascular surgeons are suggested. For centers that perform lead extractions in children or young adults, pediatric cardiologists as well as pediatric electrophysiologists should also be included. In section 8.1 "Cardiovascular Implantable Electronic Device Infection") an evaluation by physicians with specific expertise in cardiovascular implantable electronic devices (CIED) infection and lead extraction is recommended for patients with documented (class I recommendation, level of evidence $\mathrm{C}$ ) and suspected (class IIa recommendation, level of evidence C) CIED infection [6]. Putting the given information together with the assumption that in most cases, worldwide, the primary operator is an electrophysiologist or cardiologist [7], the suggested Heart Team for lead extraction procedures is represented by the following specialists: electrophysiologist or cardiologist, cardiothoracic surgeon, interventional radiologist, vascular surgeon, infectious disease specialist (in CIED infection cases) and pediatric cardiologist/ electrophysiologist (in children/young adults).

In order to evaluate current opinions and real-world practice among lead extraction experts a short online survey was created and distributed among a pool of lead extraction specialists who were participants of the International Lead Extraction Expert Meeting (ILEEM) 2018.

\section{Methods}

A short survey was created by formulating 10 questions: 9 closed-ended questions (2 dichoto- mous question [22.2\%], 7 multiple choice questions [77.8\%]) and 1 open-ended question (question on country of work) (Table 1). 7 of the $9(77.8 \%)$ closed-ended questions had the additional option to enter details on not available answer items ("other"). The goal was to generate relevant questions that could be answered in less than $5 \mathrm{~min}$, in order to get a maximum response rate. The invitation to participate was sent out by email amongst lead extraction specialists who were recruited from the participant pool of the ILEEM, which is held annually in Berlin, Germany. The recipients were encouraged to forward the invitation to other lead extraction practitioners.

The survey was performed by using an online tool called SurveyMonkey (www.surveymonkey. com; SurveyMonkey Inc., San Mateo, California, USA).

\section{Statistics}

Answers were analyzed with the tool provided by SurveyMonkey (www.surveymonkey.com; SurveyMonkey Inc., San Mateo, California, USA). Categorical variables are presented as numbers and percentages.

\section{Results}

The invitation to participate was sent to 48 lead extraction specialists by email with a collector link to an internet-based survey at the end of October 2018. The survey was closed at the end of November 2018 and 43 answers to the survey were recorded, a return rate of $89 \%$. The average time spent for completing the survey was $2 \min 38 \mathrm{~s}$.

\section{Participant countries}

Forty one participants of this survey were located in 16 different countries with the following distributions of answers: Austria (3), Czech Republic (1), Denmark (1), Finland (1), Germany (2), Italy (2), Japan (3), Netherlands (1), New Zealand (1), Poland (11), Spain (2), Sweden (1), Switzerland (4), Thailand (1), United Kingdom (3), United States (4). Two respondents did not answer country of origin.

\section{Annual volume of TLE procedures}

This question was answered by all participants. The detailed answers are shown in Figure 1. More than 30 procedures per year, being a common definition of high-volume centers, were performed in $83.7 \%$ of centers. 
Table 1. Questions of the online International Lead Extraction Expert Meeting (ILEEM) survey on the Heart Team approach in lead extraction procedures.

\begin{tabular}{|c|c|}
\hline $\begin{array}{l}\text { Questions of ILEEM survey on Heart Team approach in lead } \\
\text { extraction procedures }\end{array}$ & Question type \\
\hline 1. In which country are you working? & Open-ended \\
\hline 2. How many TLE procedures are performed in your clinic per year? & Closed-ended (multiple choice) \\
\hline 3. Where are TLE procedures performed in your hospital? & Closed-ended (multiple choice) \\
\hline 4. Who is predominantly performing TLE procedures in your hospital? & Closed-ended (multiple choice) \\
\hline 5. Who is part of your team performing TLE procedures? & closed-ended (multiple choice) \\
\hline 6. Who is performing TEE during TLE procedures in your hospital? & Closed-ended (multiple choice) \\
\hline $\begin{array}{l}\text { 7. How is cardiac surgical backup for TLE procedures organized } \\
\text { in your hospital? }\end{array}$ & Closed-ended (multiple choice) \\
\hline $\begin{array}{l}\text { 8. Would you consider an "Extended Heart Team Approach" as beneficial } \\
\text { for the treatment quality of patients requiring TLE procedures? }\end{array}$ & $\begin{array}{l}\text { Closed-ended } \\
\text { (dichotomous question) }\end{array}$ \\
\hline $\begin{array}{l}\text { 9. In your opinion who should be the members of an "Extended Heart } \\
\text { Team" for TLE procedures? }\end{array}$ & Closed-ended (multiple choice) \\
\hline 10. Do you perform team trainings with your TLE team? & Closed-ended (dichotomous ques \\
\hline
\end{tabular}

All closed-ended multiple choice questions had the additional option to enter details on not available answer items ("other"). TLE — transvenous lead extraction; TEE — transesophageal echocardiography

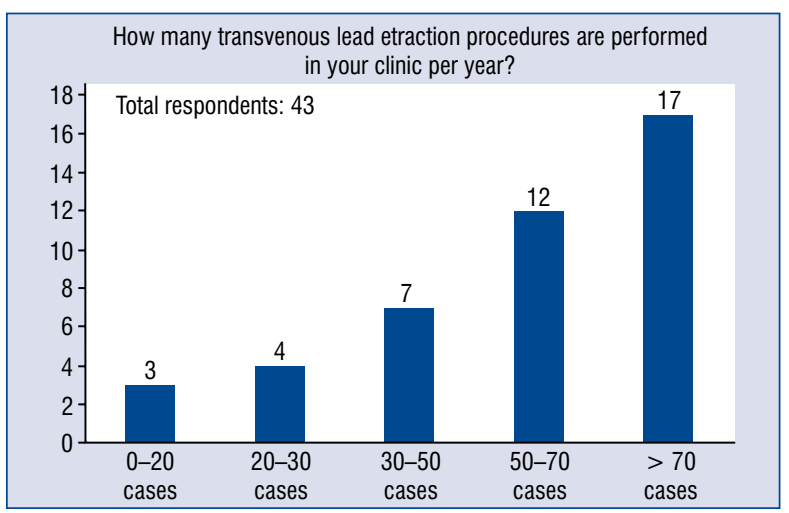

Figure 1. Annual center volume of transvenous lead extraction procedures amongst survey participants.

\section{Procedural environment}

The answer rate on this question was $100 \%$. The detailed answers are shown in Figure 2. Eighty-six percent of all participants performed lead extraction procedures in an operating room (OR), with most procedures done in a hybrid OR. Two answers were "other" and specified as OR and a mixture of OR and hybrid OR.

\section{Primary operator in lead extraction procedures}

This answer was completed by 42 participants. The primary operator is an electrophysiologist in $24(57.1 \%)$ centers, a cardiologist in $10(23.8 \%)$

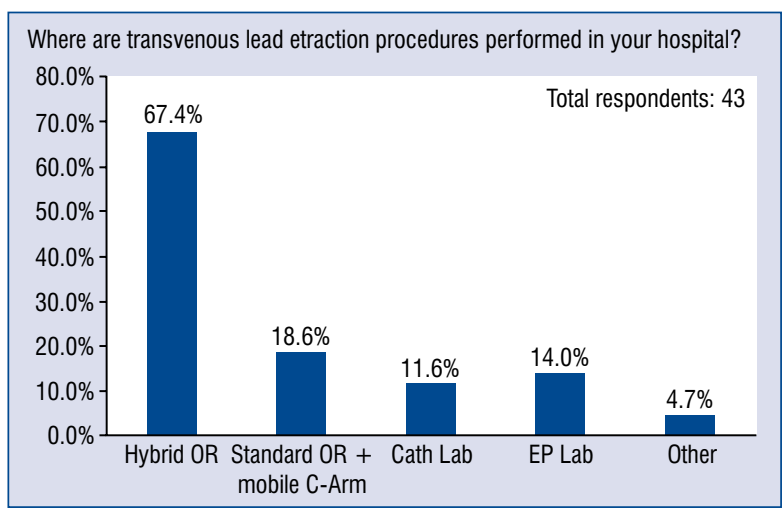

Figure 2. Procedural environment of transvenous lead extraction procedures. (Multiple responses were allowed. Percentages calculated in relation to the total number of respondents); OR - operating room.

centers and a cardiac surgeon in $8(19.1 \%)$ centers. Five additional comments were given mainly stressing the presence of a cardiac surgeon when the procedure is performed by an electrophysiologist or cardiologist.

\section{Current team composition in lead extraction procedures}

All participants answered this question. The detailed answers are shown in Figure 3. Given answers were physicians (cardiologist, electrophysiologist, cardiac surgeon, anesthesiologist) as 


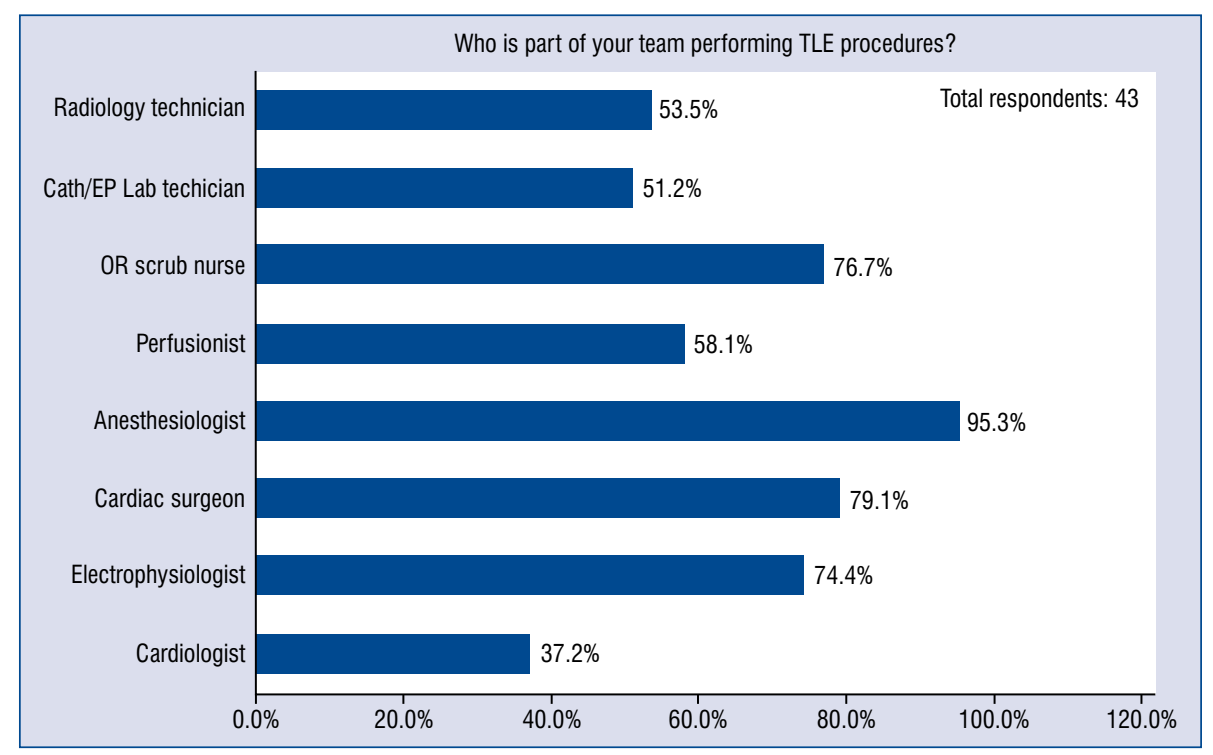

Figure 3. Current team members of lead extraction teams. (Multiple responses were allowed. Percentages calculated in relation to the total number of respondents); OR - operating room; TLE — transvenous lead extraction.

well as non-physician members (perfusionist, OR scrub nurse, Cath/EP Lab technician, radiology technician).

\section{Performance of TEE during lead extraction procedures}

This answer was completed by 42 participants. In most cases transesophageal echocardiography (TEE) is performed by an anesthesiologist ( $\mathrm{n}=25$; $59.5 \%)$. In 17 (40.5\%) centers TEE was performed by a cardiologist. Two $(4.8 \%)$ centers have a specific echocardiography technician for this task. In almost $10 \%$ (4 centers; $9.5 \%$ ) TEE was not routinely performed during TLE procedures. In $1(2.4 \%)$ center, intracardiac echocardiography (ICE) was used as an ultrasound monitoring tool during TLE procedures.

\section{Cardiac surgical backup}

Forty-one participants answered this question. In all of the responses, participating centers had cardiac surgical backup available and present but with differing access and extent. Detailed results are shown in Table 2.

\section{Extended Heart Team approach in TLE procedures}

To collect the opinions of participants on an "extended heart team approach" in TLE procedures, the following question was posed: "Would you consider an "Extended Heart Team Approach"
Table 2. Cardiac surgical backup during transvenous lead extraction procedures (participants: 43, answered: 41, skipped: 2).

\begin{tabular}{lc}
\hline Cardiac surgical backup & Responses \\
\hline $\begin{array}{l}\text { Cardiac surgeon scrubbed and } \\
\text { present during the procedure }\end{array}$ & $17(41.5 \%)$ \\
$\begin{array}{l}\text { Cardiac surgeon in the operating } \\
\text { room - not scrubbed }\end{array}$ & $13(31.7 \%)$ \\
$\begin{array}{l}\text { Cardiac surgeon in the hospital } \\
\text { No cardiac surgeon available }\end{array}$ & $11(26.8 \%)$ \\
\hline
\end{tabular}

as beneficial to the quality of treatment of patients requiring TLE procedures?". This question was answered by $100 \%$ of participants.

Thirty-seven participants in the survey $(86.0 \%)$ considered the extended Heart Team approach as beneficial, whereas for $6(14.0 \%)$ answered that it was not considered as beneficial.

\section{Members of an "Extended Heart Team" for TLE procedures}

This answer was completed by 41 participants. Besides the electrophysiologist (80.5\%) and the cardiologist (48.8\%), the cardiac surgeon (95.1\%), the anesthesiologist $(90.2 \%)$ and the infectious disease specialist $(78.0 \%)$ were considered important members of an extended lead extraction heart team. Detailed results are shown in Figure 4. 


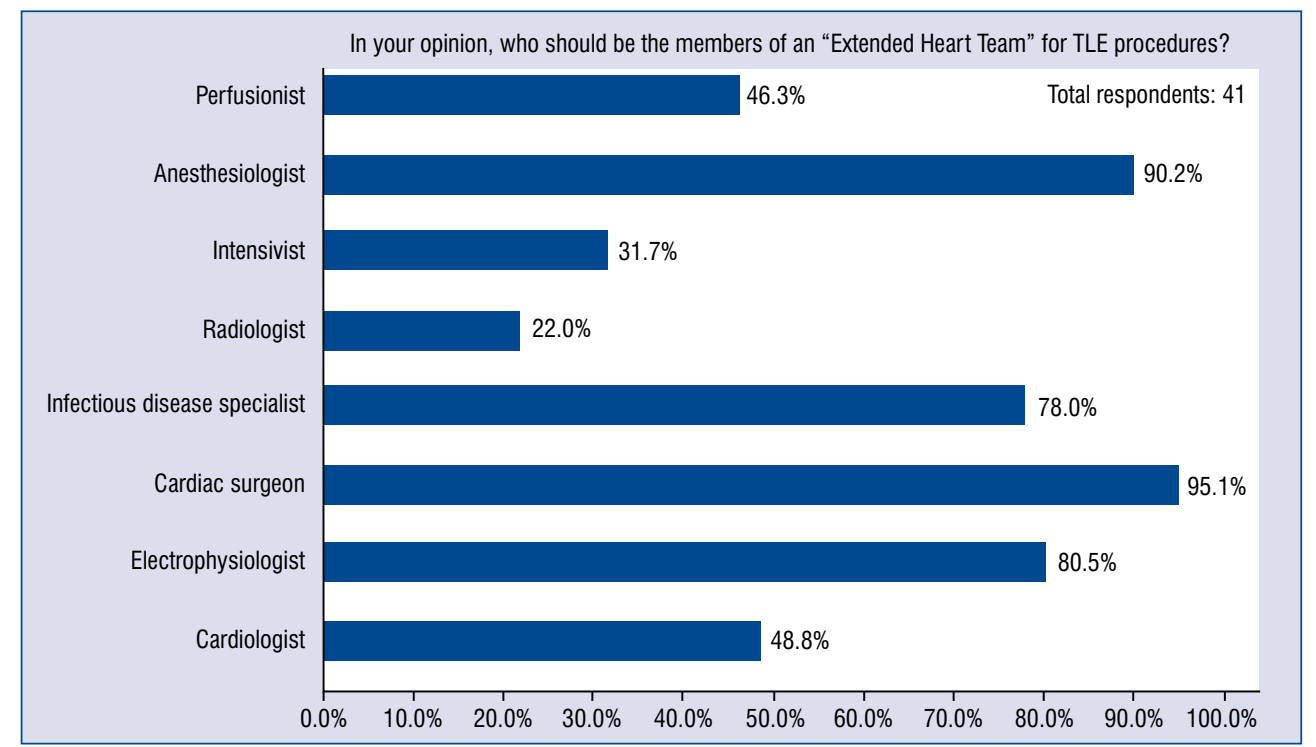

Figure 4. Opinion of the survey participants on the composition of an extended Heart Team in transvenous lead extraction (TLE) procedures. (Multiple responses were allowed. Percentages calculated in relation to the total number of respondents).

One respondent was uncertain about the terminology of an "Extended Heart Team", highlighting the potential for a broad team definition or composition. Additional potential members such as an echocardiography specialist were suggested. The quality, experience and skill mix of the team membership was also considered to be important.

\section{Training TLE teams}

This question was answered by $100 \%$ of the survey participants. $48.8 \%$ (21) of the centers perform specific team training with their TLE team, whereas $51.2 \%$ currently do not perform team training.

Additional information was supplied by 15 respondents. These comments included reporting the frequency of trainings as monthly, quarterly, twice annually or when a new member enters the team. Format of training was comprised of: workshops, seminars, clinical conferences, emergency procedure training, review of techniques, external trainings and simulation.

\section{Discussion}

A multidisciplinary team approach is now considered an integral part of current methods for providing patient-centered therapy under many cardiovascular conditions. In patients with complex coronary artery disease, it was shown that the decision-making process in a Heart Team is reproducible and that outcomes are successfully implemented in a majority of cases $[8,9]$.

The 2017 European Society of Cardiology guidelines on the management of valvular diseases recommended the concepts of a Heart Team approach and establishment of heart valve centers. Requirements of a heart valve center include a multidisciplinary team which meet on a regular basis, work with standard operating procedures (SOP), have the availability of multiple high-quality imaging techniques, conduct regular consultations with extracardiac departments and other hospitals, have the availability of back-up services and implement data reviews [1].

Given the results of this survey on the current composition of Heart Teams in lead extraction procedures and that a majority of participants regard the extended Heart Team approach to be beneficial to the quality of treatment of patients, the requirements for a Heart Team approach in lead extraction procedures can be summarized as similar to those for valvular heart disease: regular meetings, SOP-based approaches, availability of imaging specialists, infectious disease specialists as well as intensivists, close contact with referring non-extraction centers and implementation of data reviews for quality assurance purposes (Table 3 ).

The composition of the Heart Team is an important aspect. Based on the results of this survey, physicians of different specialties 
Table 3. Requirements for a Heart Team approach in lead extraction procedures.

Requirements for a lead extraction Heart Team
approach
Regular meetings
Standard operating procedures-based approaches
Availability of specialists:
- imaging specialists/radiologists
- infectious disease specialists
- intensivists
Close contact to referring non-extraction centers
Implementation of data review for quality assurance
purposes

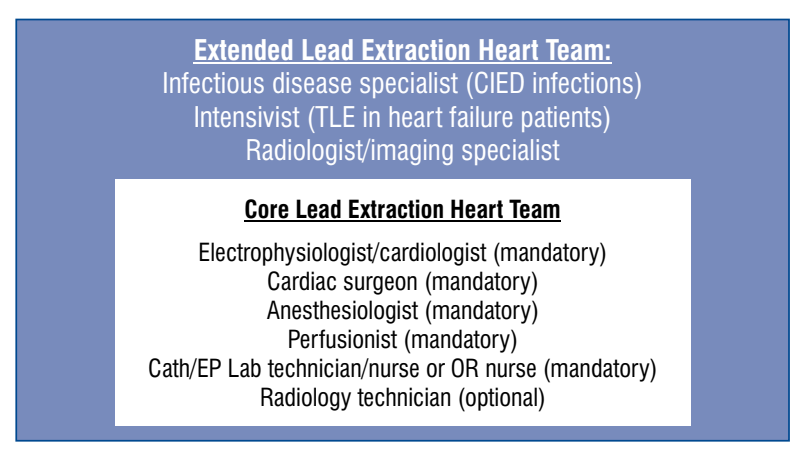

Figure 5. Concept of a core and an extended lead extraction Heart Team approach.

as well as non-physician members should be members of a lead extraction Heart Team. The definition or composition was deliberately not defined in the survey, in order to draw comment and not to influence answers. Based on the results of this survey we propose the concept of a combined core and extended Heart Team in TLE procedures (Fig. 5). The core lead extraction Heart Team should consist of all professionals involved in the actual lead extraction procedure: electrophysiologist/cardiologist (mandatory), cardiac surgeon (mandatory), anesthesiologist (mandatory), perfusionist (mandatory), Cath/EP Lab technician/ nurse or OR scrub nurse (mandatory) and radiology technician (optional). The extended Heart Team in TLE procedures additionally consists of the following professionals: Infectious diseases specialist (in cases with documented or suspected CIED infection), intensive care specialists (especially in TLE procedures in heart failure patients or when sepsis or multiorgan support is required), radiologist/imaging specialist (when special imaging modalities are required pre-operatively). A wider membership is important when considering the pre- and post-procedure management and does not exclude the need for consultation outside of the group. The role of the group should not be restricted to the performance of the procedure alone, but to be involved in pre-, peri- and postprocedure management.

The composition of the core TLE Heart Team is already well accepted in most centers. The concept of an extended lead extraction Heart Team still needs to be established and defined amongst the wider clinical cardiology community. It is a fact that performing lead extraction procedures at specialized lead extraction centers leads to higher procedure volume and better patient outcomes, with a well-documented volume-outcome relationship for lead extraction procedures [7, 10]. The two most common causes of non-procedure related inhospital mortality in the ELECTRa registry were sepsis and heart failure. In this registry, amongst others, predictors of increased all-cause mortality during hospitalization were found to be systemic infection (odds ratio 4.93, 95\% confidence interval 2.72-8.93) and New York Heart Association class III/IV (odds ratio 4.08, 95\% confidence interval 2.24-7.43) [7]. Consequently, regular involvement of specialist physicians for the treatment of septic complications or heart failure makes good clinical sense in order to improve outcomes for these subgroups of lead extraction patients. This makes the infectious disease specialist service of particular importance for patients with CIED infections (especially systemic infections). For heart failure patients, especially for those having cardiac resynchronization systems extracted, the intensivists and/or heart failure teams have important roles in helping manage and improve post-procedure survival. Furthermore, the availability of extracorporeal life support and short-term mechanical circulatory support may be beneficial for selected heart failure patients. In certain patients special imaging techniques may be required to confirm a suspected diagnosis (e.g. FDG/PET CT scan for suspected pocket infection), to assess special anatomical situations (e.g. CT angiography for venous occlusion or CT scan to confirm inadvertently placed leads in the left ventricle) or to assess lead course in relation to critical anatomical structures (e.g. superior vena cava, tricuspid valve) $[11,12]$. The vast majority (86\%) of the survey participants considered an extended Heart Team approach as beneficial for improving the quality of TLE procedural planning and performance. 
Last but not least, this survey revealed that approximately half of the centers contacted $(48.8 \%)$ undertook team training. Furthermore, the responses show a large heterogeneity in terms of the frequency and format of such team training. This is probably due to the fact that training teams specifically in lead extraction procedures remains in its infancy. There is considerable surgical team training predominantly for the non-technical skill sets that have been adopted in many centers which is applicable and transferable. Since many TLE procedures are performed in hybrid surgical environments, team training routines already exist for the more generic tasks. Specific team training for TLE work should be considered since the volume of procedures for all team members may be small in comparison to other work performed (other cardiac surgical procedures, electrophysiology procedures). Possible targets for team trainings are rehearsals of uncomplicated lead extraction procedures to improve familiarization and situational awareness with such work as well as crisis management for peri-procedural complications and management of postoperative care. With regard to procedural task training (performance of the procedure and management of complications) the use of virtual reality (VR) or augmented reality (AR) techniques may be beneficial but are as yet unproven and not specifically available for TLE procedures. Besides being used in training scenarios these VR and AR technologies may also be used as an adjunct for optimizing procedure planning and rehearsal prior to the case. These are important areas for future development.

\section{Limitations of the study}

This survey provides a consensus group opinion from a select group of clinicians who are or have been participants of the ILEEM in Berlin, Germany (www.ileem.com). Therefore, the survey participants do not represent an average group of physicians in real world practice, especially with regard to low and medium volume extraction centers. All participants perform TLE procedures using a variety of tools and techniques with $83.7 \%$ of all participants undertaking more than 30 TLE procedures annually, a common definition for a high-volume center [7]. Almost $40 \%$ of the contacted centers performed more than 70 procedures per year, highlighting the expertise amongst respondents to this survey. Only lead extraction experts involved with ILEEM provided opinions which may not reflect the experience and opinions of others, e.g. in low volume centers or views in other geographical regions. The survey was designed to gain an understanding of current opinions about the importance of a team approach to TLE procedures and provoke further discussion within the community.

The impact on clinical outcomes of such an extended Heart Team approach has to be investigated in future studies as a verification of this current expert opinion on the benefits of this approach backed by solid data.

\section{Conclusions}

This survey supports the importance of establishing lead extraction Heart Teams in specialized lead extraction centers to potentially improve patient outcomes. The concept of a core and an extended Heart Team approach in lead extraction procedures was introduced. The clinical benefits have to be proven in future studies.

\section{Conflict of interest: None declared}

\section{References}

1. Baumgartner H, Falk V, Bax JJ, et al. ESC Scientific Document Group. 2017 ESC/EACTS Guidelines for the management of valvular heart disease. Eur Heart J. 2017; 38(36): 2739-2791, doi: 10.1093/eurheartj/ehx391, indexed in Pubmed: 28886619.

2. Neumann FJ, Ahlsson A, Alfonso F, et al. 2018 ESC/EACTS Guidelines on myocardial revascularization. Eur Heart J. 2018; 34(10): 2449-2496, doi: 10.1093/eurheartj/ehy394/5079120.

3. Kirchhof P, Benussi S, Kotecha D, et al. 2016 ESC Guidelines for the management of atrial fibrillation developed in collaboration with EACTS. Eur J Cardiothorac Surg. 2016; 50(5): e1-e88, doi: 10.1093/ejcts/ezw313, indexed in Pubmed: 27663299.

4. Ponikowski P, Voors AA, Anker SD, et al. ESC Scientific Document Group. 2016 ESC Guidelines for the diagnosis and treatment of acute and chronic heart failure: The Task Force for the diagnosis and treatment of acute and chronic heart failure of the European Society of Cardiology (ESC)Developed with the special contribution of the Heart Failure Association (HFA) of the ESC. Eur Heart J. 2016; 37(27): 2129-2200, doi: 10.1093/ eurheartj/ehw128, indexed in Pubmed: 27206819.

5. Habib G, Lancellotti P, Antunes M, et al. 2015 ESC Guidelines for the management of infective endocarditis. Eur Heart J. 2015; 36(44): 3075-3128, doi: 10.1093/eurheartj/ehv319.

6. Kusumoto FM, Schoenfeld MH, Wilkoff BL, et al. 2017 HRS expert consensus statement on cardiovascular implantable electronic device lead management and extraction. Heart Rhythm. 2017; 14(12): e503-e551, doi: 10.1016/j.hrthm.2017.09.001, indexed in Pubmed: 28919379.

7. Bongiorni MG, Kennergren C, Butter C, et al. ELECTRa Investigators. The European Lead Extraction ConTRolled (ELECTRa) study: a European Heart Rhythm Association (EHRA) Registry of Transvenous Lead Extraction Outcomes. Eur Heart J. 2017; 38(40): 2995-3005, doi: 10.1093/eurheartj/ehx080, indexed in Pubmed: 28369414. 
8. Pavlidis AN, Perera D, Karamasis GV, et al. Implementation and consistency of Heart Team decision-making in complex coronary revascularisation. Int J Cardiol. 2016; 206: 37-41, doi: 10.1016/j. ijcard.2016.01.041, indexed in Pubmed: 26774827.

9. Sanchez CE, Dota A, Badhwar V, et al. Revascularization heart team recommendations as an adjunct to appropriate use criteria for coronary revascularization in patients with complex coronary artery disease. Catheter Cardiovasc Interv. 2016; 88(4): E103-E112, doi: 10.1002/ccd.26276, indexed in Pubmed: 26527352.

10. Deshmukh A, Patel N, Noseworthy PA, et al. Trends in Use and Adverse Outcomes Associated with Transvenous Lead Removal in the United States. Circulation. 2015; 132(25): 2363-2371, doi: 10.1161/CIRCULATIONAHA.114.013801, indexed in Pubmed: 26534954.

11. Vatterott P, Syed I, Khan A. Lead extraction imaging. Cardiac Electrophysiol Clin. 2018; 10(4): 625-636, doi: 10.1016/j. ccep.2018.07.007.

12. Ahmed FZ, James J, Cunnington C, et al. Early diagnosis of cardiac implantable electronic device generator pocket infection using ${ }^{18}$ F-FDG-PET/CT. Eur Heart J Cardiovasc Imaging. 2015; 16(5): 521-530, doi: 10.1093/ehjci/jeu295, indexed in Pubmed: 25651856. 